\title{
Utilizando recursos das tecnologias da informação e comunicação para apoio ao ensino da disciplina expressão gráfica nos cursos de engenharia
}

\section{Using information technologies and communication resources at graphic expression teaching in the enginnering courses}

\author{
Carlos Vitor de Alencar Carvalho ${ }^{1}$ \\ Janaina Veiga ${ }^{2}$ \\ Júlio César da Silva ${ }^{3}$

Palavras-chave:

Software Educativo

Educação Matemática

Geometria Descritiva

Desenho Geométrico

\section{Resumo:}

Este trabalho apresenta reflexões da utilização de recursos das Tecnologias da Informação e Comunicação - TIC - no apoio ao ensino da disciplina de expressão gráfica nos cursos de Engenharia. A disciplina expressão gráfica tem no seu conteúdo tópicos de desenho técnico e de geometria descritiva. A proposta apresenta a utilização das TIC em dois momentos: um com o uso do software GeoDescritiva e outro com a utilização de animações geradas especificamente para o curso a partir do software livre Régua e Compasso. A utilização dessa metodologia foi aplicada com alunos de cursos de engenharia elétrica, ambiental e de produção. Ao final do artigo são apresentados e discutidos os resultados do uso dessa metodologia em sala de aula.

\section{Abstract}

This work presents reflection about the use of information technologies and communication resources - TIC - as support to the teaching at the discipline graphic expression in the engineering courses. The subject graphic expression has geometric drawing content and descriptive geometry in its topics. The proposal presents the use of TIC in two moments: one with the use of the software GeoDescritiva and other one with the use of animations specifically generated for the course starting by the free software Compass and Ruler. The use of this methodology was applied with students at electric engineering, environmental engineering and production engineering. The results of the use of this methodology at the classroom are presented and discussed at the end of this article.
\end{abstract}

Key words:

Educational Software

Mathematics Education

Descriptive Geometry

Technical Drawing

\footnotetext{
1 Docente do Centro Universitário de Volta Redonda - UniFOA (Volta Redonda - RJ). Docente do Programa de Pós-Graduação - Mestrado Profissional em Educação Matemática da Universidade Severino Sombra - USS (Vassouras - RJ). Docente do Instituto Superior de Tecnologia de Paracambi.

${ }^{2}$ Docente do Programa de Pós-Graduação - Mestrado Profissional em Educação Matemática da Universidade Severino Sombra - USS (Vassouras - RJ)

${ }^{3}$ Docente do Programa de Pós-Graduação - Mestrado Profissional em Educação Matemática da Universidade Severino Sombra - USS (Vassouras - RJ). Docente do Centro Universitário Serra dos Órgãos - UNIFESO (Teresópolis - RJ). Docente do Instituto Superior de Tecnologia em Ciências da Computação - IST-Rio (Rio de Janeiro - RJ)
}

Recebido em 06/2011

Aprovado em $08 / 2011$

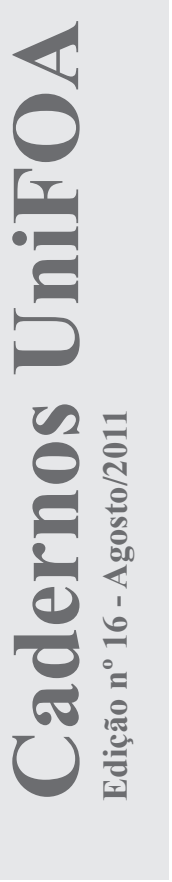




\section{Introdução}

A utilização das Tecnologias da Informação e Comunicação (TIC) como apoio aos processos de ensino e de aprendizagem não pode mais se ignorada pelos educadores e estabelecimentos de ensino, uma vez que tais tecnologias são instrumentos que possuem um grande potencial pedagógico para os professores. Com elas as distâncias diminuem, as informações caminham mais rapidamente permitindo uma ampliação e integração do conhecimento e conseqüentemente uma mudança de postura dos educadores.

Isto torna inegável a importância do debate sobre as TIC e seus impactos sobre os processos de construção do conhecimento. Neste sentido o Professor Nelson Pretto (2006:18), um dos referencias teóricos desde trabalho, destaca que "[...] no campo das instituições escolares precisamos refletir sobre o fazer pedagógico para conhecermos como os envolvidos estão recebendo a influência tecnológica e como reagem no seu dia a dia. A realidade que se apresenta constituiu um desafio para a escola como um todo". As escolas do século XXI têm que adotar uma nova metodologia em sala de aula na qual a tecnologia esteja englobada, entretanto, para que a escola mude, é necessário que exista uma nova concepção de construção do conhecimento, uma nova forma de conceber escola. Uma escola que prepara o aluno para uma sociedade informatizada. Outro referencial teórico deste trabalho, Borba \& Penteado (2001) afirma que o acesso a escola deve ser visto como um direito e que todos os alunos, sejam de escolar particular ou pública, devem ter no atual momento, mínimo, uma "alfabetização tecnológica", que não devem ser como um curso de informática, mas como uma nova mídia.

Especificamente no ensino da engenharia o uso das TIC é uma realidade em suas diversas subáreas, como estruturas, elétrica, geotécnica, hidráulica, etc. Na literatura encontramse alguns exemplos como em Carvalho et al. (2008) (Figura 1) que apresenta um software para estudo de mecanismos de transportes de contaminantes.

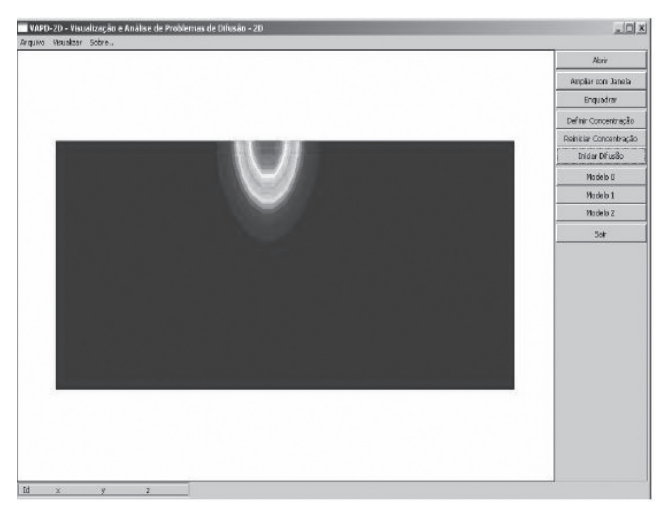

Figura 1 - Simulador VAPD-2D para apoio ao ensino de processos de transporte por difusão.

Em Guillermo et al. (2005) são apresentados simuladores computacionais na área de hidráulica e em Carvalho et al. (2005) (Figura 2) é apresentado um simulador gráfico e interativo para estudo de conforto em ambientes construídos muito utilizado por estudantes e profissionais de arquitetura. Em Carvalho \& Carvalho (2009) é apresentado outro exemplo sobre um software educacional para o estudo de tensões através do Círculo de Mohr (Figura 3).

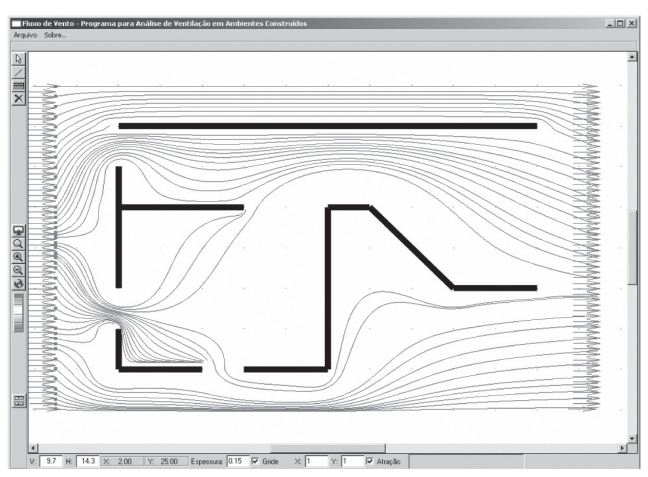

Figura 2 - FLUXOVENTO - Visualização de linhas de fluxo em ambientes construídos.

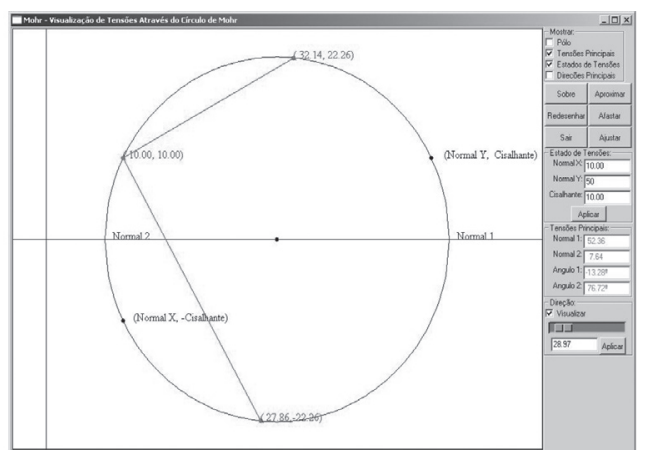

Figura 3 - Estudo do estado de tensões através do círculo de Mohr.

Em todos os exemplos citados acima o ponto comum é a visualização. Ela é importante para o entendimento do fenômeno que está sendo estudado, estimulando a intuição e a percepção dos estudantes. 
Fischbein (1990) ao analisar a visualização, afirmou que freqüentemente o conhecimento intuitivo é identificado com a representação visual. Tendemos a pensar que aquilo que não conseguimos imaginar visualmente é difícil de perceber mentalmente. A representação visual contribui para a organização da informação em representações sinópticas e desta assim, constitui um fator para o entendimento do problema que está sendo trabalhado. Uma imagem visual organiza os dados em estruturas significativas, tornando-se fator importante na orientação do desenvolvimento analítico de uma solução. No caso da disciplina expressão gráfica, a visualização é um fator muito importante para o entendimento dos conteúdos.

Basicamente, no estudo da disciplina expressão gráfica são envolvidos conceitos de desenho técnico e geometria descritiva. O desenho técnico é responsável pela definição das formas, dimensões e posição dos objetos utilizados e necessários para a engenharia e arquitetura. A geometria descritiva, cujas origens remetem ao método criado por Gaspard Monge, tem o objetivo de representar de uma maneira coerente e sem dupla interpretação, objetos tridimensionais em um plano bidimensional.

Fazendo uma revisão na literatura, podem-se verificar alguns exemplos do uso de TIC para estudo do desenho técnico e geometria descritiva. Por exemplo, em Bolgheroni et al. (2008) é apresentado um estudo caso do uso de TIC no ensino de desenho técnico e em Morgado et al. (2002) apresenta o desenvolvimento de um software para o estudo da geometria descritiva e suas potencialidades.

Com base nestas reflexões, a proposta deste trabalho é apresentar o uso de TIC para apoio ao ensino da disciplina de expressão gráfica. A metodologia aborda não somente a utilização do software GeoDescritiva em sala de aula, desenvolvido pelos autores deste trabalho, mas também a utilização de animações desenvolvidas pelos autores com temas sobre desenho técnico usando o software livre Régua e Compasso (RÉGUA E COMPASSO, 2009), sendo esta integração o diferencial das abordagens encontradas na literatura. A metodologia proposta permite aos estudantes visualizar conceitos e, junto com o professor, experimentar hipóteses em um mesmo ambiente, propiciando ambiente de investigação lúdico e significativo.

\section{Metodologia}

As atividades propostas neste trabalho foram realizadas em alunos do primeiro ano de cursos de Engenharia Ambiental e Elétrica. A abordagem inicial foi através de questionários (entrevistas) como técnica privilegiada de comunicação.

Durante as aulas teóricas foram apresentadas e disponibilizadas aos alunos o software GeoDescritiva e animações feitas através do software Régua e Compasso como indica o diagrama da Figura 4. A seguir serão detalhadas as informações da cada uma das ferramentas utilizadas em sala de aula.

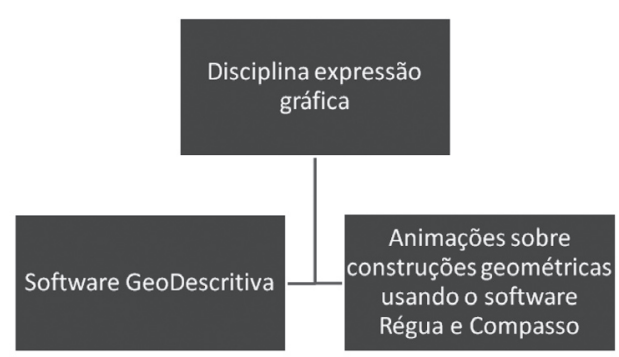

Figura 4 - Estrutura adotada na utilização das TIC na disciplina de expressão gráfica.

\subsection{Animações usando o software Régua e Compasso}

Como dito anteriormente, animações foram elaboradas usando o software Régua e Compasso e todo o processo de gravação foi feito através do software AutoScreenRecorder 3.0 versão freeware (AUTOSCREENRECORDER, 2009). As animações foram geradas no formato de extensão avi, e contemplam os seguintes temas:

- Construção de retas perpendiculares;

- Construção de triângulos;

- Construção de retas paralelas;

- Construção de concordâncias;

- Traçado de bissetriz;

- Divisão de segmentos.

Todas as animações foram gravadas para a geração e produção de um CD-ROM chamado "Animações sobre Desenho Técnico" (Figura 5, Figura 6 e Figura 7). A Figura 8 mostra um exemplo da animação gerada. O aluno pode verificar e reverificar a sua construção contri- 
buindo para o entendimento do assunto. Todas as animações geradas estão disponíveis em Carvalho (2011a, 2011b, 2011c, 2011d, 2011e, 2011f, 2011g, 2011h, 2011i, 2011j, 2011k).

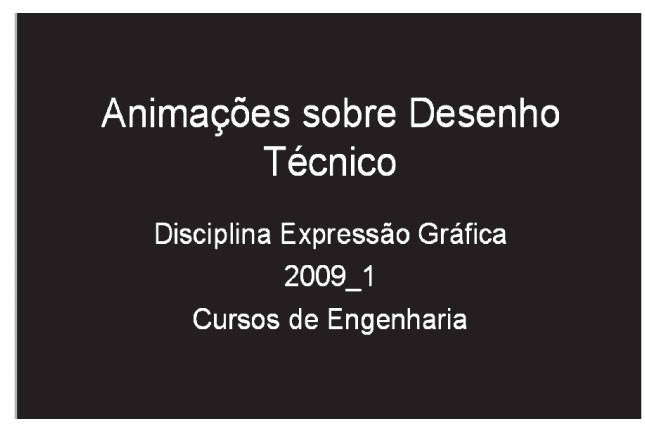

Figura 5: Tela inicial do CD-ROM de animações.

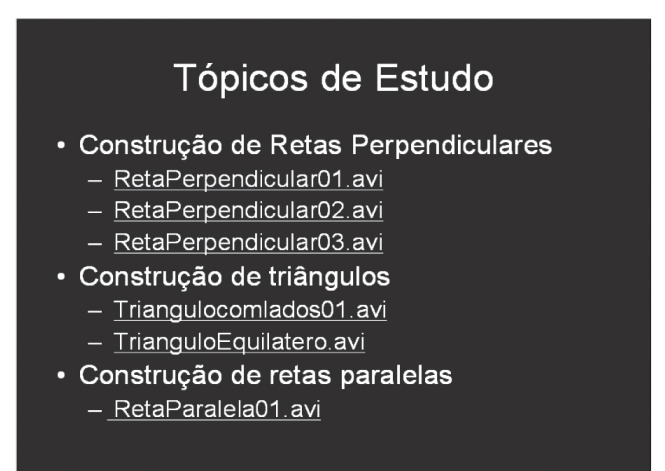

Figura 6: Tópicos de estudo. Os vídeos podem ser visualizados através de hiperlink em cada item.

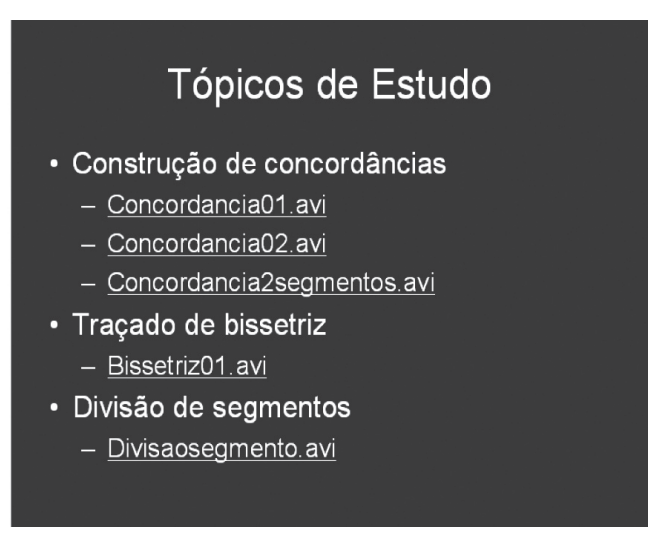

Figura 7: Tópicos de estudo. Os vídeos podem ser visualizados através de hiperlink em cada item.

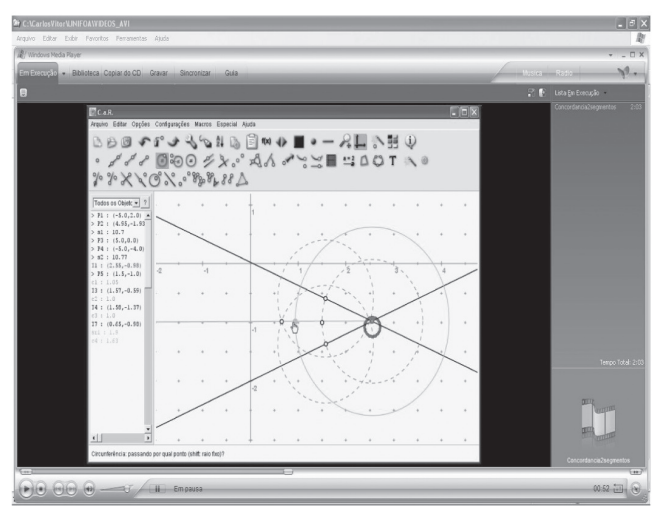

Figura 8: Animação sobre concordância. O aluno pode observar a construção feita no software Régua e Compasso verificando suas particularidades.

\subsection{O Software GeoDescritiva}

A geometria descritiva foi um método desenvolvido por Monge no século XVIII, e tornou-se uma importante metodologia para a formação profissional dos engenheiros. Entretanto, o ensino e o entendimento dos conceitos da geometria descritiva, para os alunos iniciantes, são um grande desafio. A compreensão do método, apesar da sua eficiência, requer muito treinamento e exercícios progressivos e sistematizados. Esta foi uma das principais motivações para o desenvolvimento do software GeoDescritiva que se encontra disponível no website (Figura 9) indicado em Carvalho (20111).

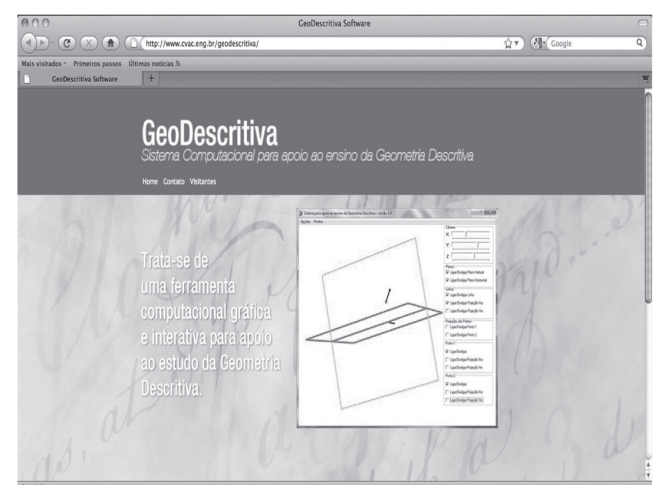

Figura 9 - Website do software GeoDescritiva.

O software foi desenvolvido usando o ambiente Delphi (CORNELL, 1995) e o sistema gráfico OpenGL (COHEN \& MANSSOUR, 2006). O GeoDescritiva permite a visualização tridimensional dos planos: vertical e horizontal (Figura 10). Na mesma figura é possível observar a interseção dos planos, chamada linha de terra. Na Figura 11 é possível identificar os diedros que são os quadrantes formados pelos planos.

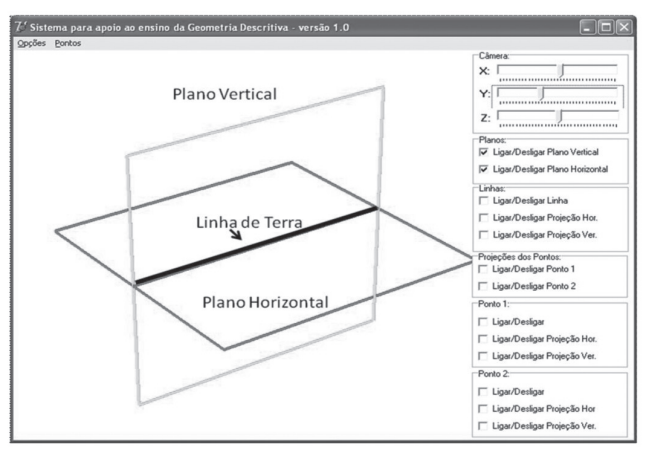

Figura 10: Interface do sistema GeoDescritiva. 


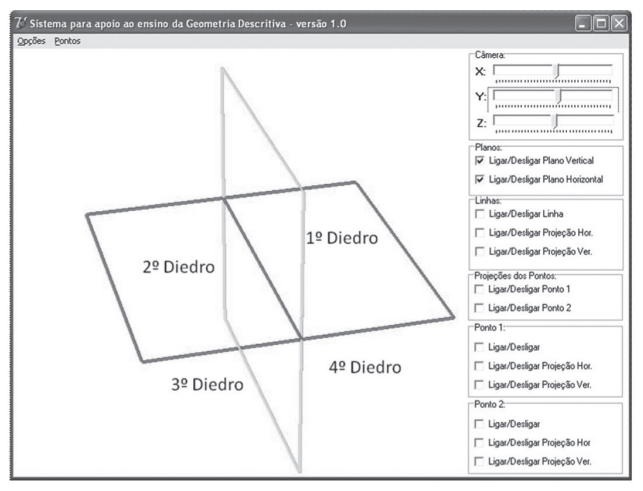

Figura 11: Diedros formados pelos planos: vertical e horizontal.

O software possui um menu no lado esquerdo onde é possível, utilizando os deslizadores, rodar os planos nos três eixos coordenados ( $x, y$ e $z$ ), além de visualizar ou não a reta e pontos projetos nos planos: vertical e horizontal. A partir desses conceitos iniciais o professor pode iniciar os estudos sobre ponto e reta.

A Figura 12 mostra as características das projeções de um ponto no plano vertical superior e plano horizontal posterior. Quando o plano vertical é rebatido no sentido anti-horário, ele coincide com o plano horizontal, formando, o que tecnicamente, se chama épura. Nela é possível representar um ponto ou figura do espaço pelas suas projeções no plano. A Figura 13 apresenta a épura como exemplo do ponto $[-3,-4,-5]$, que poderá ser desenhada pelo professor usando o quadro negro, uma vez que nesta versão ainda não está disponível a construção da épura, sendo este um dos objetivos das implementações para as futuras versões.

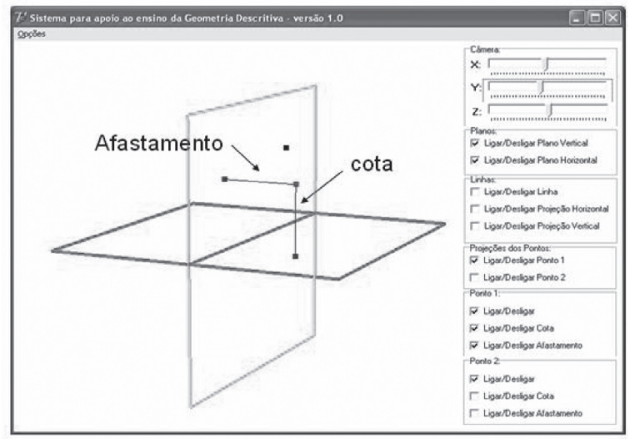

Figura 12: Característica de um ponto no espaço: seu afastamento e sua cota.

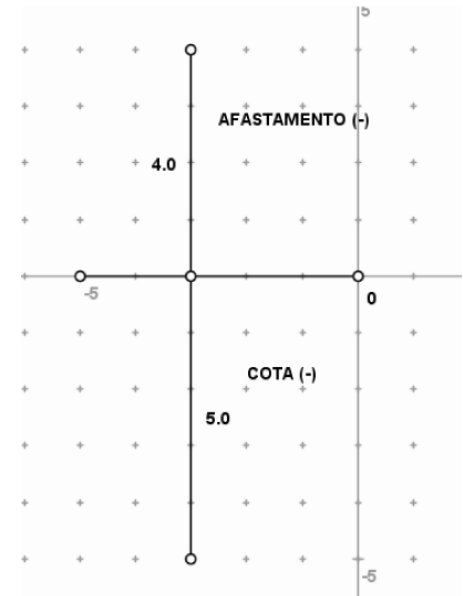

Figura 13: Exemplo de épura do ponto $[-3,-4,-5]$.

\subsection{Utilizando o software em sala de aula}

Após a apresentação do software e das animações para a turma, algumas atividades foram sugeridas. Primeiramente os alunos, após a visualização das animações em sala de aula, foram estimulados a desenvolver as mesmas construções usando papel, lápis e os instrumentos régua e compasso. Em seguida com o software GeoDescritiva foram propostas as seguintes atividades:

a. Definir dois pontos no $1^{\circ}$ diedro especificando sua cota, afastamento e abscissa. O sistema disponibiliza uma interface para tal tarefa (Figura 14).

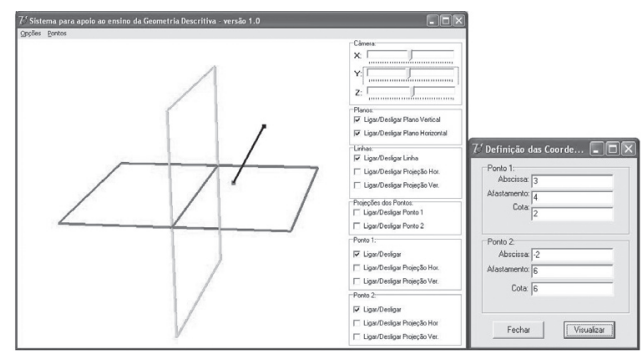

Figura 14: Definição de dois pontos para compor uma reta no primeiro diedro.

b. Ligar as projeções (Figura 15) e verificar sua visualização e no caderno desenvolver a épura da reta construída.

C. Repetir os processos para pontos no $2^{\circ}, 3^{\circ}$ e $4^{\circ}$ diedros. 


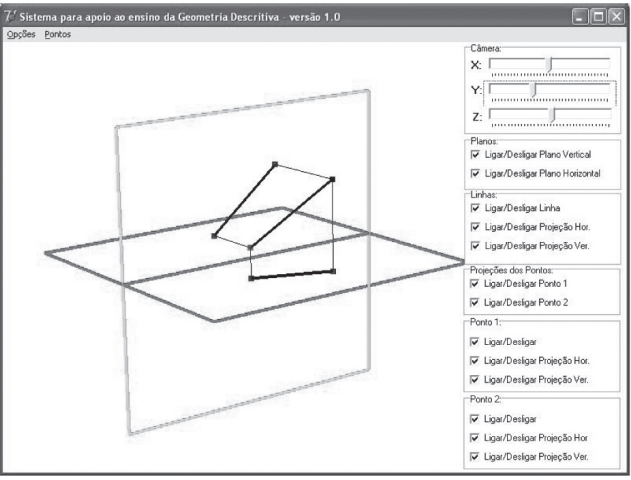

\section{Resultados e Discussões}

O questionário (Quadro 1) visava observar a aceitação, pelos discentes, do uso de novas tecnologias em sala de aula e da metodologia proposta para a disciplina de expressão gráfica.

Figura 15: Projeções do ponto 1, ponto 2 e da reta no plano vertical e horizontal.

Quadro 1: Questões proposta para avaliação do software em sala de aula.

\begin{tabular}{c|l}
\hline $\begin{array}{c}\text { Número da } \\
\text { questão }\end{array}$ & \multicolumn{1}{c}{ Questão } \\
\hline 1 & Como você avalia de forma geral o uso de tecnologias em sala de aula? \\
\hline 2 & $\begin{array}{l}\text { A metodologia de mostrar os conceitos da disciplina usando o software e animações foi } \\
\text { bem aceita por você? }\end{array}$ \\
\hline 3 & $\begin{array}{l}\text { Você acha que o software e as animações são ferramentas que ajudam no processo de } \\
\text { ensino-aprendizagem das construções geométricas? }\end{array}$ \\
\hline 4 & $\begin{array}{l}\text { Para você foi importante o uso de novas tecnologias para apoio ao processo de ensino- } \\
\text { aprendizagem? }\end{array}$ \\
\hline 5 & O quanto você aprendeu com a utilização de tais recursos em sala de aula? \\
\hline
\end{tabular}

Os estudantes consideram positivo (com valor máximo de $57.1 \%$ considerando excelente) o uso de novas tecnologias em sala de aula (Tabela 2 e Figura 16 - primeira questão). Após as atividades propostas continuaram avaliando positivamente o uso das novas tecnologias (Tabela 3 e Figura 17 - quarta questão).

Em relação à metodologia, houve uma aceitação acima da média do uso dos softwares e animações que pode ser observado nas respostas da segunda e terceira questões (Tabela 4 e Figura 18 - segunda questão; Tabela 5 e Figura 19 - terceira questão). Em relação à aprendizagem em ambas as turmas mais de um terço da turma (67\% e $60.6 \%$ ) consideraram que aprenderam muito com a metodologia utilizadas nas aulas. (Tabela 6 e Figura 20 quinta questão).

Tabela 2 - Dados da Figura 16.

\begin{tabular}{|l|c|c|c|c|c|}
\hline & Excelente (\%) & Bom (\%) & Regular (\%) & Fraco (\%) & Insuficiente (\%) \\
\hline Eng. Ambiental & 57,1 & 42,9 & 0 & 0 & 0 \\
\hline Eng. Elétrica & 15,2 & 48,5 & 30,3 & 3 & 3 \\
\hline
\end{tabular}

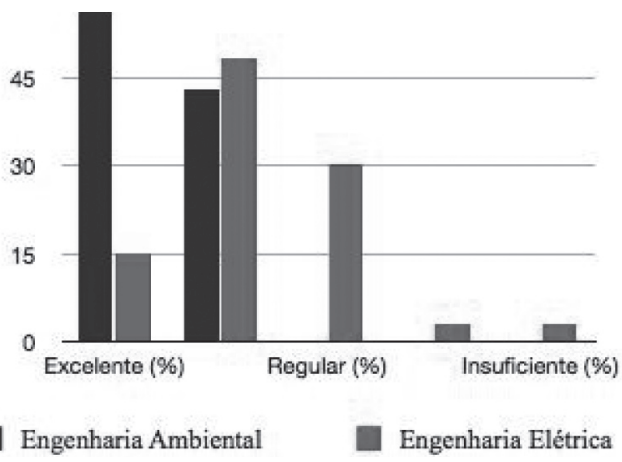

Figura 16: Gráfico dos dados obtidos para a primeira questão. 


\begin{tabular}{|l|c|c|}
\hline & Sim (\%) & Não (\%) \\
\hline Eng. Ambiental & 100 & 0 \\
\hline Eng. Elétrica & 54,5 & 45,5 \\
\hline
\end{tabular}

\begin{tabular}{|l|c|c|c|}
\hline & $\begin{array}{c}\text { Muito } \\
(\%)\end{array}$ & $\begin{array}{c}\text { Razoável } \\
(\%)\end{array}$ & $\begin{array}{c}\text { Pouco } \\
(\%)\end{array}$ \\
\hline Eng. Ambiental & 3,6 & 64,3 & 32,1 \\
\hline Eng. Elétrica & 18,2 & 42,4 & 39,4 \\
\hline
\end{tabular}

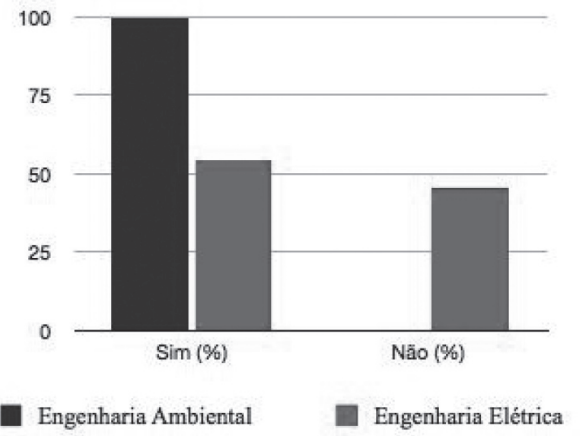

Figura 17: Gráfico dos dados obtidos para a segunda questão.

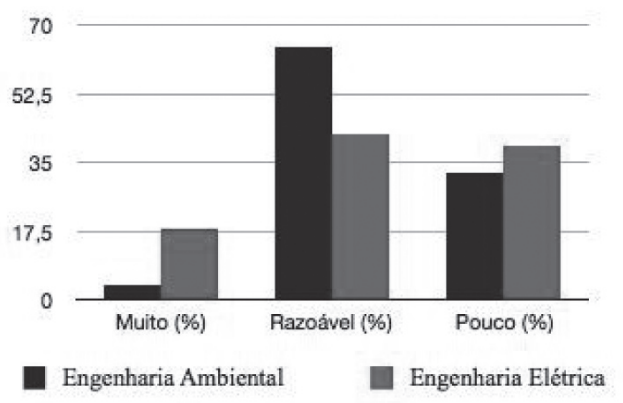

Figura 20: Gráfico dos dados obtidos para a quinta questão.

Tabela 4 - Dados da Figura 18.

\begin{tabular}{|l|c|c|}
\hline & Sim (\%) & Não (\%) \\
\hline Eng. Ambiental & 89,3 & 10,7 \\
\hline Eng. Elétrica & 60,6 & 39,4 \\
\hline
\end{tabular}

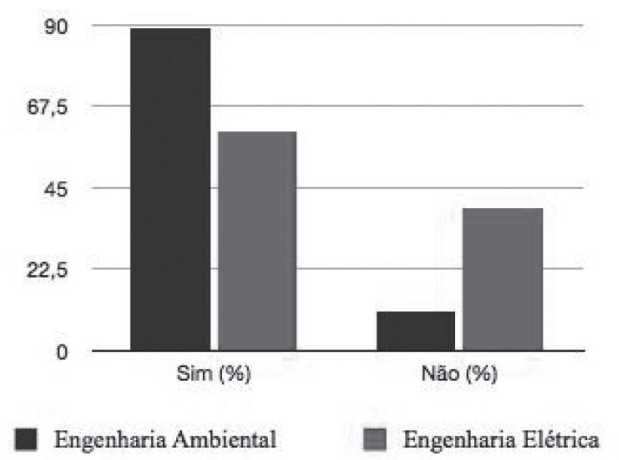

Figura 18: Gráfico dos dados obtidos para a terceira questão.

Tabela 5 - Dados da Figura 19.

\begin{tabular}{|l|c|c|c|}
\hline & $\begin{array}{c}\text { Sim } \\
(\%)\end{array}$ & $\begin{array}{c}\text { Mais ou } \\
\text { Menos (\%) }\end{array}$ & $\begin{array}{c}\text { Não } \\
(\%)\end{array}$ \\
\hline Eng. Ambiental & 89,3 & 10,7 & 0 \\
\hline Eng. Elétrica & 60,6 & 36,3 & 3,1 \\
\hline
\end{tabular}

\section{Agradecimentos}

$\mathrm{O}$ primeiro autor agradece ao $\mathrm{CNPq}$ pelo apoio financeiro através da Bolsa de Produtividade em Desenvolvimento Tecnológico e Extensão Inovadora - DT. A segunda autora agradece à FAPERJ pelo apoio financeiro através do programa "Apoio à melhoria do ensino em escolas sediadas no estado do Rio de Janeiro - 2009”. 


\section{Referências}

1. AUTOSCREENRECORDER.Disponível em: http://www.wisdom-soft.com/. Acessado em 10 de outubro de 2009. 2009.

2. BOLGHERONI, W. \& SILVEIRA, I. F Software Livre Aplicado ao Ensino de Geometria e Desenho Geométrico. Anais do XXVIII Congresso da SBC - WIE - Workshop sobre Informática na Escola, Belém, Pará, 2008.

3. BORBA, M. C. \& PENTEADO, M. G. - Informática e Educação Matemática - coleção tendências em Educação Matemática - Editora Autêntica, Belo Horizonte, Minas Gerais, 2001.

4. CARVAlho, C. V. A. Animação da construção de uma reta perpendicular - modo 2. Disponível em http://www. youtube.com/watch? $\mathrm{v}=\mathrm{Us} 9 \mathrm{JbWqDbdI}$. Acessado em 30 de maio de 2011. 2011a.

5. CARVAlHO, C. V. A. Animação da construção dos triângulos a partir dos lados. Disponível em http://www.youtube. com/watch?v=3yF9t9ImKKI. Acessado em 30 de maio de 2011. 2011 b.

6. CARVAlHO, C. V. A. Animação da concordância entre dois segmentos convergentes, onde se conhece o ponto de interseção, com um arco Disponível em: http://www.youtube.com/ watch? $\mathrm{v}=\mathrm{h} 4 \mathrm{tQUDOoH} 3 \mathrm{M}$. Acessado em 30 de maio de 2011. 2011c.

7. CARVAlHO, C. V. A. Animação da concordância entre duas retas paralelas. Disponível em: http://www.youtube.com/ watch? $\mathrm{v}=\mathrm{ndkJ} 8 \mathrm{~h} 19 \mathrm{aI} 4$. Acessado em 30 de maio de 2011. 2011d.

8. CARVAlhO, C. V. A. Animação da concordância entre uma reta e um arco Disponível em: http://www.youtube.com/ watch? $\mathrm{v}=$ OctcTjELVZI. Acessado em 30 de maio de 2011. 2011e.
9. CARVAlHO, C. V. A. Animação da construção bissetriz de um ângulo formado por dois segmentos. Disponível em http://www.youtube.com/ watch? $\mathrm{v}=$ XIuEE38j9mk. Acessado em 30 de maio de 2011. 2011 f.

10. CARVAlho, C. V. A. Animação da construção de uma reta perpendicular - modo 1. Disponível em http://www. youtube.com/watch? $\mathrm{v}=\mathrm{MTbDrkq}$ BcM. Acessado em 30 de maio de 2011. 2011g.

11. CARVAlHO, C. V. A. Animação da construção de uma reta perpendicular - modo 3. Disponível em http://www. youtube.com/watch? $\mathrm{v}=\mathrm{XshZ21Gdjak}$. Acessado em 30 de maio de 2011. 2011h.

12. CARVAlhO, C. V. A. Animação do traçado de duas tangentes à um círculo passando por ponto externo comum as duas tangentes. Disponível em: http:// www.youtube.com/watch? $\mathrm{v}=\mathrm{Wz} 4$ zxDXDYM. Acessado em 30 de maio de 2011. 2011 i.

13. CARVAlHO, C. V. A. Animação do traçado de uma tangente à um círculo. Disponível em: http:/www.youtube.com/ watch?v=6gi0deFsbA4. Acessado em 30 de maio de 2011. 2011j.

14. CARVALHO, C. V. A. Animação do traço de uma reta paralela por um ponto exterior a uma reta. Disponível em http://www. youtube.com/watch? $\mathrm{v}=6 \mathrm{gU} 1 \mathrm{ygqCj \textrm {Vw }}$. Acessado em 30 de maio de 2011. 2011k.

15. CARVAlHO, C. V. A. Software GeoDescritiva. Disponível em: http:// www.cvac.eng.br/Geodescritiva/. Acessado em 30 de maio de 2011. 20111.

16. CARVAlho, C. V. A., CARVAlHO, J. V. Um software educacional gráfico e interativo para apoio ao estudo de tensões planas através do círculo de Mohr. Revista de Informática Mater Dei, v.4, p.1-12, 2008. 
17. CARVAlHO, C. V.A., CARVALHO, J. V., SILVA, J. C. da VAPD-2D - Simulador para Apoio ao ensino da Engenharia Ambiental. Revista Eletrônica TECCEN (on-line) Disponível em: http://www.uss.br/page/ revistateccen.asp., v.1, p.1 - 8, 2008.

18. CARVALHO, C. V. A.; MARTHA, L. F.; TEIXEIRA, W. FLUXOVENTO - Um simulador gráfico interativo para o estudo de ventilação em ambientes construídos. In: VII Encontro Nacional sobre Conforto no Ambiente Construído (ENCAC), Maceió. ISBN: 85-8947812-2. Maceió, v. 1. p. 350-360, 2005.

19. COHEN, M; MANSSOUR, I. OpenGL - Uma Abordagem Prática e Objetiva. Novatec, 2006.

20. CORnEll, G., STRAIN, T. Delphi Segredos e Soluções. $1^{\text {a }}$ Ed. São Paulo: Makron Books, 1995.

21. DESLANDES, S. F., GOMES, R. Pesquisa social: teoria, método e criatividade. Maria Cecília de Souza Minayo (organizadora). 25 a edição. Revista e atualizada. - Petrópolis, RJ: Vozes, 2007.

22. FISCHBEIN, E. Psycology and mathematics In: Nesher, P. \& Kilpatrick, J. (ed.). Mathematics and cognition: a research synthesis by the international group for the psychology of mathematics education. Cambridge: Cambrige University Press, 1990. p. 1-13. ICMI Study Series, 1990.
23. GUILlERMO, O. E. P., TAROUCO, L. M. R.; ENDRES, L. A. M. O Poder das Simulações no ensino de hidráulica. RENOTE. Revista Novas Tecnologias na Educação, Porto Alegre - Brasil; v. 3, n. 1, 2005 Disponível em: http://www.cinted. ufrgs.br/renote/maio2005/index.html. Acessado em 10 de outubro de 2009.

24. MORGADO, F.; ALMEIDA, P., Ambiente Interactivo para o Ensino da Geometria Descritiva, Actas do VI Congresso Iberoamericano de Informática Educativa, Vigo, 2002.

25. PRETTO, N., Políticas Públicas Educacionaisnomundocontemporâneo. Liinc em Revista, v.2, n.1, março 2006, p.10 - 27. Disponível em: http://www. liinc.ufrj.br/revista/index-revista.htm. Acessado em 01 de outubro de 2009.

26. RÉGUA E COMPASSO, Software Régua e Compasso, Disponível em: http://www. rene-grothmann.de/. Acessado em 10 de outubro de 2009, 2009. 